Article

\title{
Evaluation of Multi-Beam Massive MIMO Considering MAC Layer Using IEEE802.11ac and FDD-LTE
}

\author{
Fumiya Muramatsu ${ }^{1}$, Kentaro Nishimori ${ }^{1, * \mathbb{C}}$, Ryotaro Taniguchi ${ }^{1}$, Takefumi Hiraguri ${ }^{2}$ \\ and Tsutomu Mitsui ${ }^{2}$ \\ 1 Graduate School of Science and Technology, Niigata University, Niigata-shi, Niigata 950-2102, Japan; \\ muramatsu@gis.ie.niigata-u.ac.jp (F.M.); taniguchi@gis.ie.niigata-u.ac.jp (R.T.) \\ 2 Faculty of Fundamental Engineering, Nippon Institute of Technology, 4-1 Gakuendai, Miyashiro-machi, \\ Minamisaitama-gun, Saitama 345-8501, Japan; hira@nit.ac.jp (T.H.); ben321@outlook.jp (T.M.) \\ * Correspondence: nishimori@ie.niigata-u.ac.jp; Tel.: +81-25-262-7431
}

Received: 26 January 2019; Accepted: 14 February 2019; Published: 18 February 2019

\begin{abstract}
Massive multiple-input multiple-output (MIMO) transmission has attracted attention as a key technology for use in fifth-generation mobile communication systems. Multi-beam massive MIMO systems that apply beam selection in analog components and blind algorithms in digital components to eliminate the requirement for channel state information have been proposed as a method for reducing overhead. In this study, we developed an adaptive modulation scheme for implementing multi-beam massive MIMO and used computer simulation to compare it with digital and analog-digital hybrid beam-forming methods. The effectiveness of the proposed system was verified in a medium access control layer based on the IEEE802.11ac and frequency division duplex-LTE representative wireless communication standards.
\end{abstract}

Keywords: massive MIMO; analog multi-beam; hybrid beam-forming; PHY layer; MAC layer

\section{Introduction}

Cellular network data traffic volumes have increased significantly with the advent of smart devices. This trend will continue to grow as Internet of Things (IoT) equipment and big-data services become more common. To meet this demand, recent research and development have focused on achieving a 20-Gbps or more standard for future wireless communication [1,2].

Multiple-input multiple-output (MIMO) systems can be used to improve the transmission rate within a limited frequency band [3]. To this end, multi-user MIMO (MU-MIMO) has been developed to enable MIMO transmission to multiple users [4]. MU-MIMO technologies have been standardized for LTE-advanced and IEEE802.11ac [5,6]. Accordingly, massive MIMO is viewed as a fifth-generation (5G) technology, and it is expected to play an important role in achieving 5G targets [1,7].

In a MIMO system, a base station (BS) acquires channel state information (CSI) between itself and a user terminal (UT) [8]. As CSI acquisition under massive MIMO involves numerous UT-BS antenna pair channels, one of the most important challenges in implementing it is the acquisition of large amounts of CSI with a small overhead. To achieve this, it is necessary to evaluate not only the physical (PHY) layer but also the medium access control (MAC) layer. The MAC layer is a communication protocol of a part of the data link layer corresponding to the second layer of the OSI reference model in IEEE802, and it is located in a layer that is one layer above the physical layer.

Implicit beamforming (BF) has been proposed as an approach for eliminating CSI feedback in MU-MIMO systems [9]. However, the communication efficiency of short-packet communication decreases even if implicit BF is applied in a massive MIMO system [10]. 
CSI estimation and initial UT tracking, which require a significant amount of overhead, are necessary in hybrid BF massive MIMO systems [1]. A hybrid BF approach referred to as multi-beam massive MIMO transmission has been proposed to solve this problem. CSI estimation is unnecessary in this approach. The effectiveness of multi-beam massive MIMO has been demonstrated through computer simulation [11-13]. In multi-beam massive MIMO systems, a number of analog multi-beams are created for initial UT tracking and a subset of these beams with high received power are selected for use. As multi-beams have narrow beam widths, interference signals can be mitigated and residual interference can be cancelled by applying a blind array algorithm to digital beam components using only the information pertaining to a received signal [14-16]. This paper proposes a multi-beam massive MIMO approach that achieves high transmission efficiency and flexible communication using asynchronous UT transmission. As the proposed system does not require timing synchronization among UTs, all UTs can freely transmit signals while avoiding collision between their signals. The diminished signal transmission time resulting from this configuration reduces the total power consumption by the UTs.

In our previous studies, we proposed multi-beam massive MIMO beam-selection methods [13] that utilized received-signal information such as the power difference and amplitude correlation between beams. This approach was capable of appropriately performing beam-selection at a high signal-to-interference noise ratio (SINR). Furthermore, this method could be performed through signal processing using a simple configuration and was highly suitable for hybrid analog-digital massive MIMO.

Channel quality indicator (CQI) values, which can serve as an index of modulation scheme determination, cannot be obtained under multi-beam massive MIMO because it does not perform CSI estimation. In this paper, we propose a simple adaptive modulation scheme for multi-beam massive MIMO transmission based on amplitude correlation and received power. Under this method, an appropriate modulation scheme can be simply determined based on the relationship between amplitude correlation and the SINR. We assessed the proposed method by evaluating the transmission rates it achieved using the IEEE802.11ac and frequency division duplex (FDD)-LTE standards. In addition, we evaluated the throughput of the method under the MAC layer of each standard to consider overhead.

The rest of this paper is organized as follows: The general massive MIMO transmission approach is described in Section 2. In Section 3, we describe the use of amplitude correlation for modulation and propose a simple adaptive modulation scheme for multi-beam massive MIMO. In Section 4, we present our simulation model and describe its application in evaluating simple adaptive modulation schemes. Then, we describe the results of the performance evaluation of the proposed multi-beam massive MIMO method through the computer simulation of the IEEE802.11ac and FDD-LTE standard environments.

\section{Massive MIMO Transmission and Proposed System}

The bandwidth required by 5G systems is achieved using frequencies of $20 \mathrm{GHz}$ or higher. Massive MIMO utilizes BF technology to resolve propagation loss, which is one of the more serious problems encountered under high-frequency bands. Typically, a technique known as digital BF (DBF) is adopted, as shown on the right-hand side of Figure 1. In this technique, weight values are calculated through digital signal processing. However, the massive MIMO systems that apply DBF experience problems with respect to power consumption and implementation cost $[1,17,18]$.

The left-hand side of Figure 1 shows the configuration of a typical hybrid BF massive MIMO with sub-arrays [19]. In the figure, $N_{L}$ and $N_{K}$ indicate the number of elements and receivers in a sub-array, respectively. This configuration produces a hybrid analog-digital BF that tracks a desired signal through analog control while removing interference using digital signal processing, and it has attracted significant interest [20-22]. Massive MIMO is known to be highly effective in addressing the power consumption and implementation cost problems owing to its hybridization of analog and digital approaches. 
Multi-beam massive MIMO eliminates the requirement for CSI estimation by forming numerous narrow directional beams and spatially separating the signals of each UT. As a result, the synchronization between a BS and a UT, which is required for CSI estimation, becomes unnecessary. Under standard MU-MIMO, UTs must transmit signals to a BS simultaneously to employ CSI feedback prior to communication. As multi-beam massive MIMO does not require CSI feedback, a UT can communicate with a BS at any time.

Figure 2 shows the configuration of analog multi-beam massive MIMO under uplink communication, along with its 16 multi-beam pattern. The proposed system generates $N$ orthogonal multiple beams in the analog component and uses a butler matrix circuit to achieve multi-beam formation [23,24]. The butler matrix circuit, which was introduced in [24], forms an orthogonal beam using a Fast Fourier Transform (FFT) on a spatially arranged array. This system is also effective from the viewpoint of the pilot contamination problem in the multi-cell scenario because of the signals by multiple UTs can be received without the CSI estimation by the multi-beam circuit.
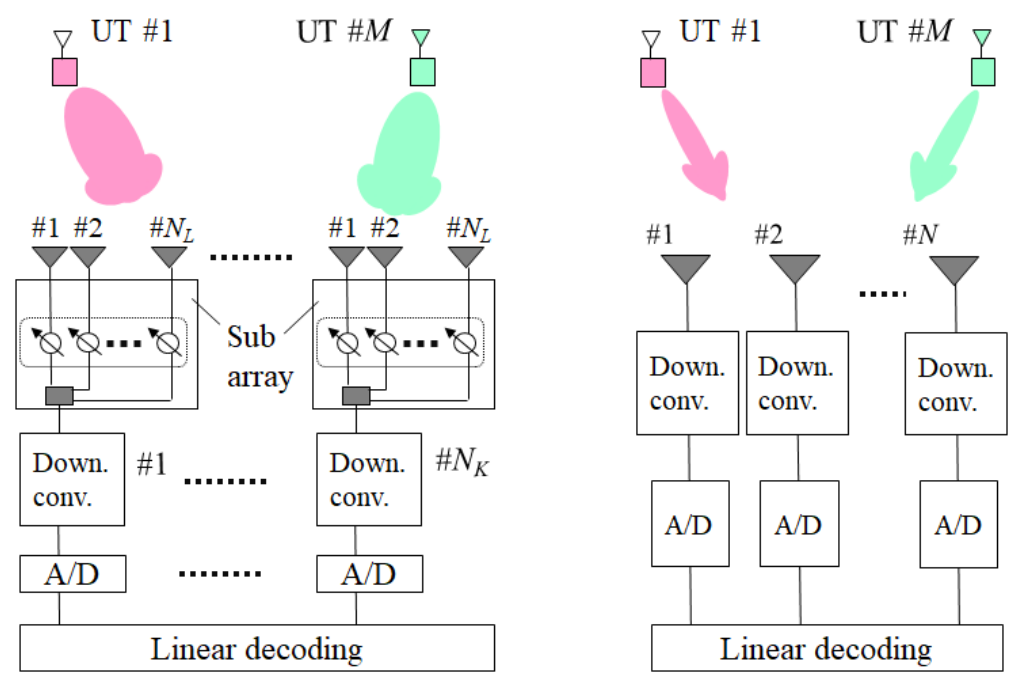

Figure 1. Uplink configurations of typical analog-digital hybrid (left) and full-digital (right) beamforming (BF) massive multiple-input multiple-output (MIMO) systems.
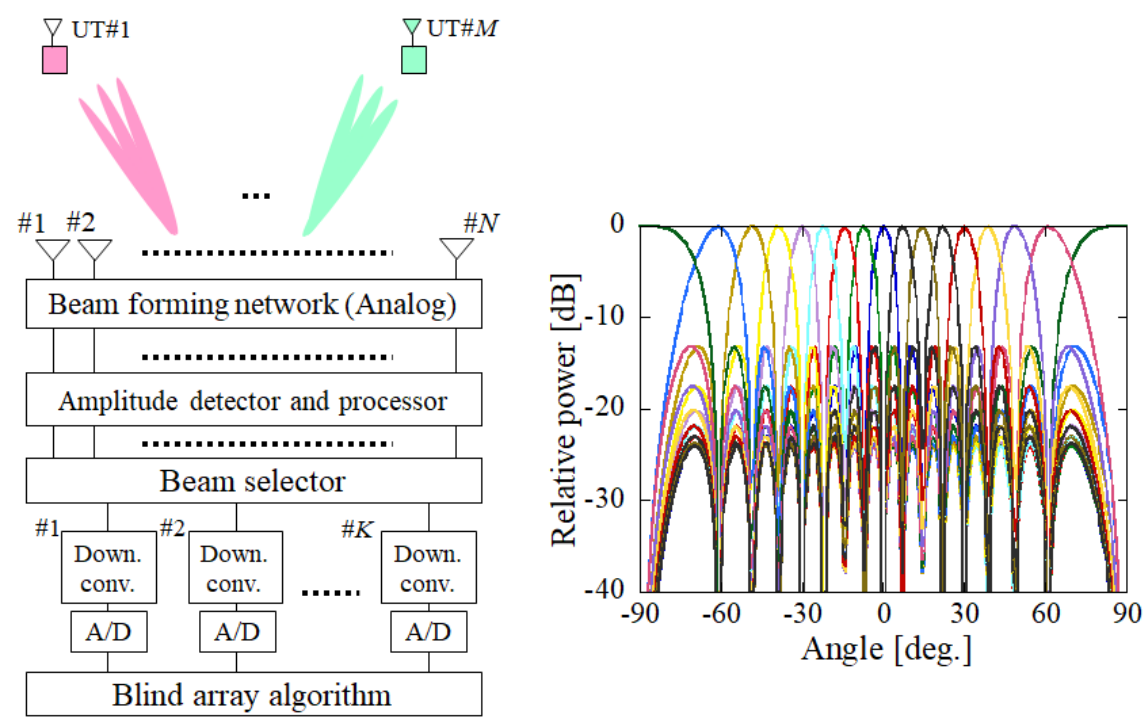

Figure 2. Uplink configuration of multi-beam massive MIMO in uplink and 16 multi-beam pattern. 
The proposed method performs beam selection using an amplitude detector and a processor. Through beam selection, it is possible to spatially separate a specific UT using beam directivity. However, interference signals from the other UTs, which are also received by side lobes, cannot be rejected through only the multi-beam forming network. A method that uses only amplitude information, such as amplitude correlation, must be used to achieve beam selection with low interference power.

The system developed in this study applies robust independent component analysis (ICA) using a blind adaptive algorithm [16,25]. ICA is a blind signal processing technique that is commonly used in image processing and medicine. In this technique, an observed random vector is decomposed into statistically independent variables [16]. ICA does not require CSI because it utilizes only received signals. The use of ICA allows for a hybrid configuration for efficient transmission in massive MIMO systems in which multi-beams are applied to analog components and a blind algorithm is applied to digital components.

\section{Adaptive Modulation for Multi-Beam Massive MIMO}

As discussed in the previous section, multi-beam massive MIMO does not require CSI estimation, thereby eliminating the requirement for a process to obtain channel information at the BS side. As a result, it is not possible to use a modulation and coding scheme (MCS) index to combine the modulation scheme and coding rate from the CQI value, as is done under current wireless communication standards $[5,6]$. Therefore, we developed an adaptive modulation scheme based on the received power and amplitude correlation obtained from performing beam selection during uplink.

Here, we explain the beam-selection method and amplitude correlation in multi-beam massive MIMO. Beam-selection is one of the major challenges in multi-beam massive MIMO. As a directivity peak cannot be directed to a given UT, it is possible for one beam to receive signals from multiple UTs. It has been confirmed that in such situations, signal separation cannot be perfectly performed in digital components, even by a blind algorithm. To address this, the authors previously proposed a beam-selection method that utilizes the power differences between the multi-beams and correlation values obtained from amplitude information [13]. By employing this method, it is possible to achieve beam selection with a high SINR by setting thresholds.

Under the proposed beam-selection method, amplitude correlation is calculated based on the covariance between the amplitudes of received and adjacent beams. The amplitude of a received signal is defined as a vector, $x$, whose number of elements is equivalent to the lengths of all transmission-signal symbols. The correlation coefficient, $\rho(x, y)$, with respect to the signal amplitudes, $x$ and $y$, is denoted as

$$
\rho(\boldsymbol{x}, \boldsymbol{y})=\frac{\sum_{i=1}^{N}\left(x_{i}-\bar{x}\right)\left(y_{i}-\bar{y}\right)}{\sqrt{\sum_{i=1}^{N}\left(x_{i}-\bar{x}\right)^{2}} \sqrt{\sum_{i=1}^{N}\left(y_{i}-\bar{y}\right)^{2}}}
$$

where $\bar{x}$ is the mean of all elements in $x$.

Figure 3 shows the relationship between amplitude correlation and the SINR. The SINR of the received signal tends to be high when the amplitude correlation is high. Based on this finding, we examined a method of determining a simple adaptive modulation approach on the downlink using the amplitude correlation and received power obtained on the uplink. 


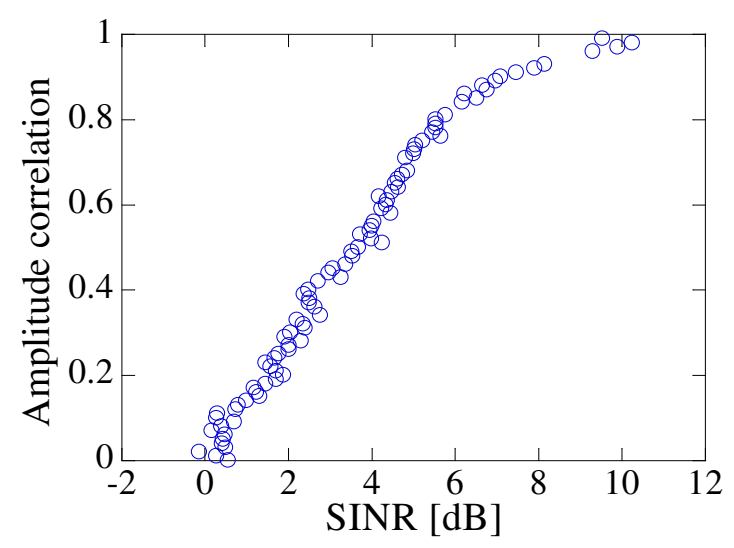

Figure 3. Relationship between amplitude correlation and signal-to-interference noise ratio (SINR).

\section{Computer Simulation}

The results of the computer simulation of the proposed system were verified in terms of the Rayleigh fading and angular spread of quadrature amplitude modulation (QAM) signals [26,27]. The simulation conditions are listed in Table 1. Figure 4 shows the simulation model, which is a scattering ring model [27] with a specific angular spread. The model applies 101 paths per UT with an angular spread of 1.0 degree. We assume flat fading in narrowband signals and do not consider delay spread. Sixty-four elements are arranged in the horizontal direction at intervals of 0.5 wavelengths, and beam width is approximately 1.60 degrees. As the assumed transmission method involves a single carrier for narrowband signals, delay waves have no influence on signal separation. The signals for each sub-carrier are regarded as narrowband signals to apply them as actual broadband signals through orthogonal frequency division multiplexing. In the UT distribution, the center direction of the BS is set as 0 degrees and individual UTs are placed at random angles within a range of -60 to 60 degrees. A constant distance is set between the BS and all UTs so that a signal-to-noise ratio (SNR) of $20[\mathrm{~dB}]$ per UT is received at the BS.

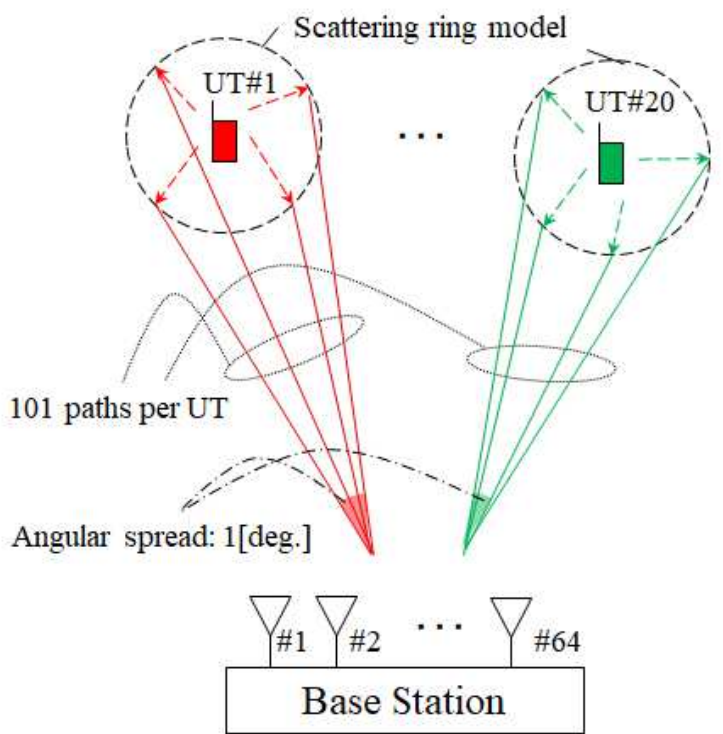

Figure 4. Simulation model. 
Table 1. Simulation conditions.

\begin{tabular}{cc}
\hline Number of receiving antennas & 64 \\
\hline Number of UTs & 20 \\
\hline Number of demodulated UTs & 8 \\
\hline MNR per UT, before array gain acquisition & $20[\mathrm{~dB}]$ \\
\hline Trial number & 4QAM (QPSK) - 1024QAM \\
\hline Data length & 3000 \\
\hline Data smoothing size & 3000 \\
\hline Number of iterations & 3000 \\
\hline
\end{tabular}

First, the processing of the uplink was simulated transmitting QPSK signals from all 20 UTs to the BS, which recorded the amplitude correlation and received power for each beam. On the downlink, the BS transmitted QPSK 1024-QAM signals to eight users with high reception power. Then, the UTs received signals from the BS calculated bit error rate (BER). We assumed that a modulation scheme yielding a BER of less than $10^{-2}$ could be applied $[28,29]$. We evaluated the applicable modulation schemes in terms of the SINR and received power recorded on the uplink to simulate an adaptive modulation scheme.

\subsection{Adaptive Modulation}

The results produced by the simulation procedure described in Section 4 are shown in Figure 5, in which the left-hand and right-hand figures show the relationship between the applicable modulation method and amplitude correlation and received power, respectively. Even though amplitude correlation increases with the modulation order, it is not appropriate to select a modulation method using only amplitude correlation because the standard deviation of the results is large. Therefore, the received power relationship shown in the right-hand figure was used to develop an indicator for determining the modulation method (Table 2). In the table, $\rho$ and $P$ represent amplitude correlation and received power, respectively.
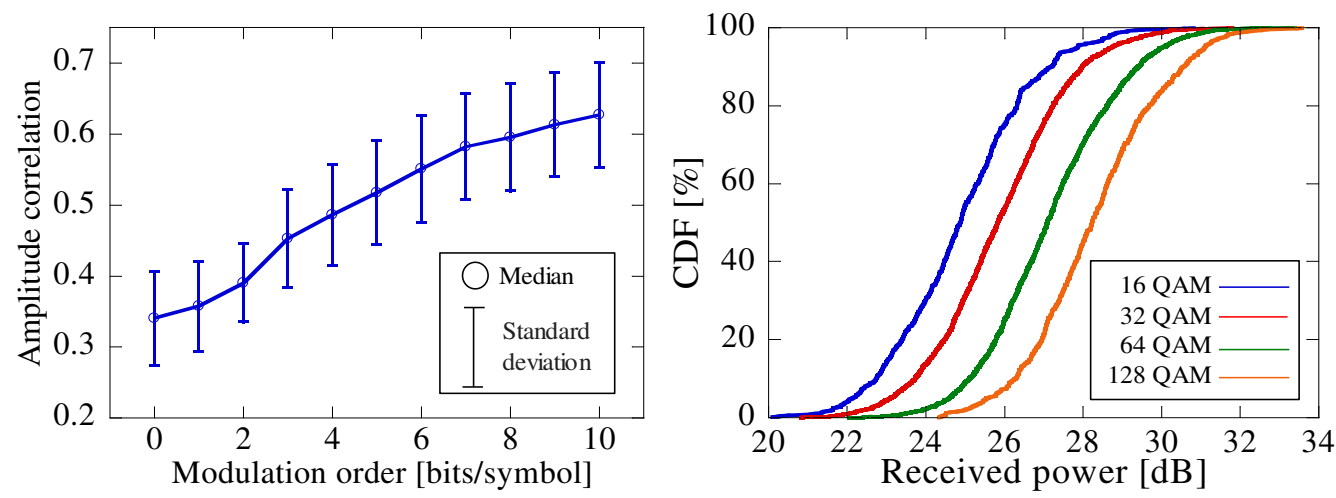

Figure 5. Relationships between applicable modulation scheme and amplitude correlation (left) and received power (right). 
Table 2. Adaptive modulation scheme for eight user terminals (UTs).

\begin{tabular}{cc}
\hline Modulation Scheme & Conditions \\
\hline None & $\rho<0.4$ \\
16-QAM & $\rho \geq 0.4$ and $P<20[\mathrm{~dB}]$ \\
32-QAM & $\rho \geq 0.4$ and $20 \leq P<24[\mathrm{~dB}]$ \\
64-QAM & $\rho \geq 0.4$ and $24 \leq P<28[\mathrm{~dB}]$ \\
128-QAM & $\rho \geq 0.4$ and $P \geq 28[\mathrm{~dB}]$ \\
\hline
\end{tabular}

\subsection{IEEE802.11ac}

We evaluated the indicator shown in Table 2 and the downlink effectiveness of multi-beam massive MIMO transmission based on the IEEE802.11ac procedures [5,30]. The simulation conditions and model were the same as those used in Section 4. Equation (2) was used as an index of evaluation based on the BER calculated on the UTs.

$$
R=\left\{\begin{array}{cl}
M(1-\mathrm{BER}) & \left(\mathrm{BER} \leq 10^{-2}\right) \\
0 & \text { (otherwise) }
\end{array}[\mathrm{bit} / \mathrm{symbol}],\right.
$$

where $M$ denotes the number of bits per symbol and $R$ denotes the number of bits per symbol obtained independently of the coding rate.

The assessment was divided into parts, i.e., the evaluation of the PHY layer without considering the overhead arising from the control signal and the evaluation of the MAC layer considering overhead. Table 3 shows the relationship between the MCS index and the transmission rate under the IEEE802.11ac 20-[MHz] operation [5]. The values of $R^{\prime}$ are obtained by multiplying the modulation order by the coding rate, and they are equivalent to $R$, which is the number of bits per symbol. Accordingly, it was assumed that an MCS index that satisfies $R \geq R^{\prime}$ could be applied to the $R$ obtained on the UTs. Based on this, we could evaluate the transmission rate corresponding to the MCS index.

Table 3. Relationship between MCS index and transmission rate under IEEE802.11ac at $20 \mathrm{MHz}$.

\begin{tabular}{ccccc}
\hline $\boldsymbol{I}_{M C S}$ & Modulation & Coding Rate & $\boldsymbol{R}^{\prime}$ [bits/symbol] & Transmission Rate [Mbps] \\
\hline 0 & BPSK & $1 / 2$ & 0.5 & 7.2 \\
1 & QPSK & $1 / 2$ & 1.0 & 14.4 \\
$\vdots$ & $\vdots$ & $\vdots$ & $\vdots$ & $\vdots$ \\
9 & 256QAM & $3 / 4$ & 6.0 & 86.7 \\
\hline
\end{tabular}

In the MAC layer, throughput is calculated according to the downlink MU-MIMO procedure shown in Figure 6 [5]. As seen in the figure, the BS first transmits request signals to all UTs to obtain channel information. Once the BS has obtained channel feedback from each UT data transmission signal, data reception processing is performed for all UTs. Throughput is obtained by dividing the data size of frame aggregation, which was set as 63,000 bytes in this evaluation, by the time required by the processes. As multi-beam massive MIMO does not require procedures such as CSI estimation or feedback, we assumed that no time other than that necessary for data transmission was required. 


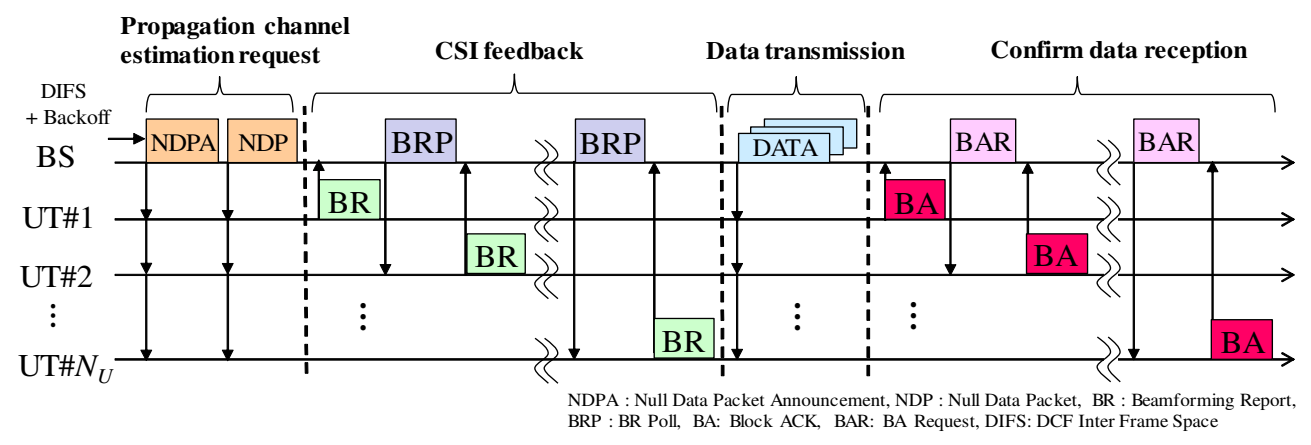

Figure 6. Downlink multi-user (MU)-MIMO procedure under IEEE802.11ac.

We evaluated the performance of a typical hybrid BF massive MIMO and compared it with those of the sub-array and full-digital BF configurations discussed in Section 2. Table 4 shows the precoding and decoding methods used under each configuration in the downlink. For the hybrid $\mathrm{BF}$, we assumed that maximum ratio combining (MRC) was performed in the analog component using a phase shifter [31]. In the digital component, the minimum mean square error (MMSE) weight calculated in the uplink was used as a precoder [31]. Eight sub-arrays $\left(N_{K}=8\right)$ and eight sub-array elements $\left(N_{L}=8\right)$ were utilized, resulting in 64 elements. In the full-digital $\mathrm{BF}$, transmission precoding was performed using a block diagonalization (BD) method with 64 elements [32].

Table 4. Precoding and decoding on downlink.

\begin{tabular}{ccc}
\hline Massive MIMO Configuration & Precoding & Decoding \\
\hline Multi-beam massive MIMO (Figure 2) & Analog multi-beam & Robust ICA \\
Hybrid BF massive MIMO (Figure 1) & MRC and MMSE & MMSE \\
Full-digital BF massive MIMO (Figure 1) & BD method & MMSE \\
\hline
\end{tabular}

Figure 7 shows the cumulative distribution function (CDF) characteristics of transmission rate and throughput. The adaptive modulation results show the characteristics obtained for each trial when the highest modulation order is selected in the modulation scheme in which the BER is $10^{-2}$ or less. The proposed modulation results are obtained using the characteristics based on Table 2 . At the assumed transmission rate, the full-digital BF configuration achieves 86.7 [Mbps], which is the maximum transmission rate obtainable under IEEE802.11ac, because it scans a sharp beam width using 64 elements. The median value of multi-beam massive MIMO is approximately 70 [Mbps], and it is asymptotic to the ideal characteristic obtained by utilizing the proposed simple adaptive modulation. The hybrid BF configuration characteristic achieves a low modulation order because beam width is widened when there are eight elements per sub-array. The full-digital configuration can achieve a throughput of only approximately 40 [Mbps] because of the overhead of channel estimation and feedback obtained when there are 64 antenna elements. In hybrid BF, overhead occurs owing to eight elements, which is the number of sub-arrays. As shown in Figure 6, throughput decreases when the transmission rate of even one UT is slow because IEEE802.11ac requires the synchronization of all UTs, as shown in Figure 6. This reduces the throughput characteristic of the hybrid BF. The multi-beam massive MIMO characteristic is the best among the three configurations because it does not require synchronization and overhead and can effectively utilize the transmission rate obtained in the PHY layer. 

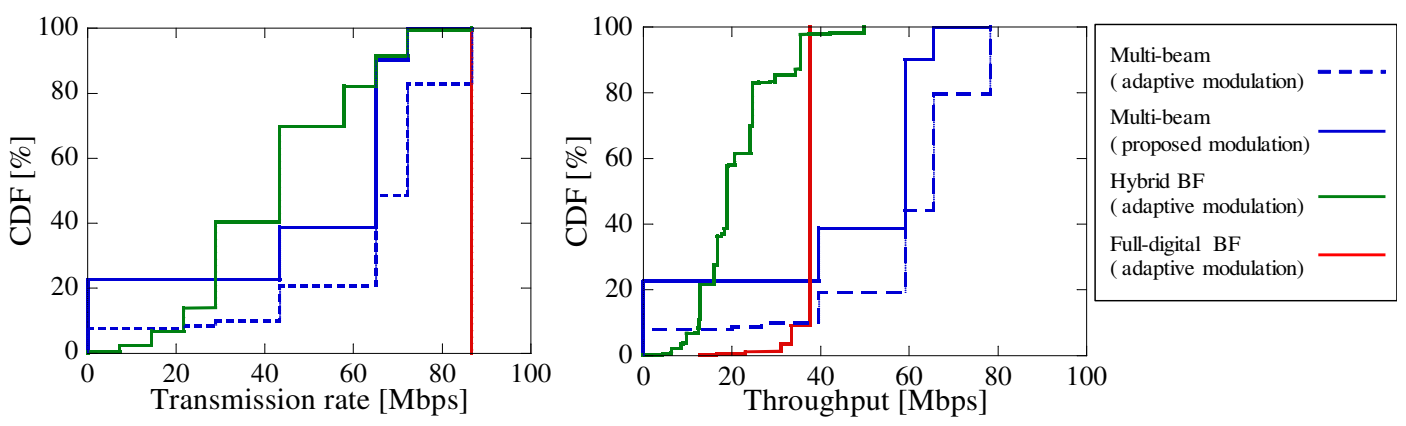

Figure 7. Characteristics of transmission rate and throughput under IEEE802.11ac.

\subsection{FDD-LTE}

We evaluated the proposed method under FDD-LTE (Release 15) [6].We assumed a bandwidth of 20 [MHz] per UT and 100 resource blocks (RBs). Table 5 shows the relationship between the MCS index and the transmission rate in LTE when there is one antenna. The transport block size (TBS) is based on [6], and the transmission rate is calculated based on the efficiency per bandwidth. The TBS corresponds to an actual data component divided into RBs and transmitted, while user data indicate the resource size for a given control signal. As in the IEEE802.11ac simulation, we compared the values of $R$ and $R^{\prime}$ produced by the respective approaches and evaluated the results in terms of the corresponding transmission rates.

Table 5. Relationship between MCS index and transmission rate under frequency division duplex (FDD)-LTE (100 RBs, $20 \mathrm{MHz}$ ).

\begin{tabular}{ccccccc}
\hline $\boldsymbol{I}_{\boldsymbol{M C S}}$ & Modulation & TBS & User Data & Coding Rate & $\boldsymbol{R}^{\prime}$ [bits/symbol] & Transmission Rate [Mbps] \\
\hline 0 & QPSK & 2792 & 30000 & 0.094 & 0.188 & 7.22 \\
1 & QPSK & 4548 & 30000 & 0.154 & 0.308 & 9.61 \\
2 & QPSK & 7224 & 30000 & 0.242 & 0.484 & 21.7 \\
& $\vdots$ & $\vdots$ & $\vdots$ & $\vdots$ & $\vdots$ & $\vdots$ \\
27 & 256QAM & 84760 & 120000 & 0.707 & 5.656 & 127.0 \\
\hline
\end{tabular}

The throughput in LTE, which is denoted here by $T$, can be calculated as follows: [6,33].

$$
\begin{aligned}
T & =T B S \times N_{\text {str }} \\
& \fallingdotseq N_{\text {subc }} \times N_{\text {slot }} \times N_{\text {sym }} \times M \times N_{\text {str }} \times C R[\mathrm{bps}],
\end{aligned}
$$

where $N_{\text {subc }}$ is the number of sub-carriers, $N_{\text {slot }}$ is the number of slots per second, $N_{\text {sym }}$ is the number of symbols per slot, $N_{\text {str }}$ is the number of streams, and $C R$ is the coding rate.

We apply Equation (3) to consider control signals because Equation (3) cannot express the number of control signals that can be eliminated under multi-beam massive MIMO.

$$
T=T B S \times \frac{\text { Userdata }^{\prime}}{\text { Userdata }} \times N_{\text {subf }} \times N_{\text {str }}[\mathrm{bps}],
$$

where $N_{\text {subf }}$ is the number of sub-frames per second. The value of Userdat $a^{\prime}$ is obtained by increasing or decreasing the number of control signals from secured Userdata, which is defined in [6]. For example, when $I_{M C S}=27$ in Table 1, Userdata is obtained as 120,000 [bits]. This corresponds to a Userdata ${ }^{\prime}$ of 128,000 [bits] in the multi-beam massive MIMO, which is obtained by removing the control signal from Userdata. As there is no complete definition of a control signal in the case of 64 elements, we assumed that one antenna port would be added to handle the additional control signal required each time the number of antennas was doubled. Therefore, Userdata' was 83,200 [bits] for 64 antennas under the full-digital configuration. 
Figure 8 shows the relationship between the transmission rate and throughput based on Equation (3). We used this figure to evaluate throughput based on overhead for each configuration.

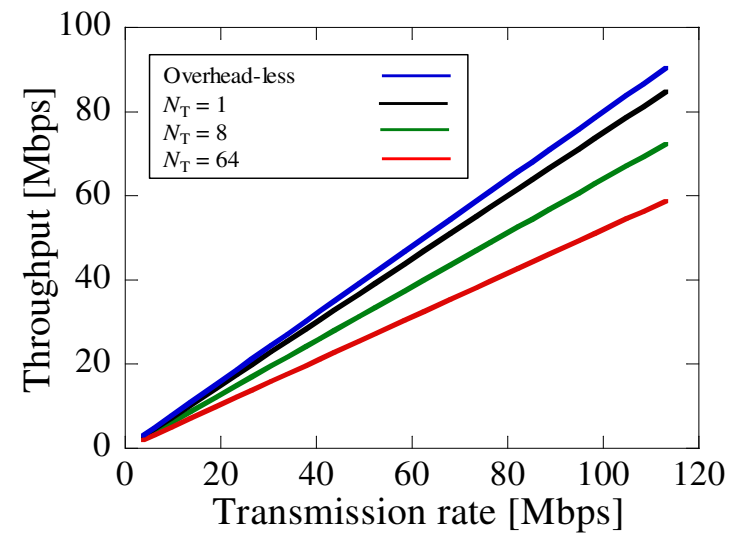

Figure 8. Transmission rate versus throughput from Equation (3).

Figure 9 shows the CDF characteristics of the transmission rate and throughput under FDD-LTE. The modulation scheme for each characteristic is determined in the same manner as that used in the evaluation under IEEE802.11ac. The transmission rate follows the same trend as that under IEEE802.11ac. However, unlike IEEE 802.11ac, a high transmission rate can be achieved even with a low modulation order. This suggests that the performance of the hybrid BF approaches that of multi-beam massive MIMO. An evaluation of throughput based on Figure 8 reveals that it increases linearly with transmission rate under all configurations. Given that multi-beam massive MIMO has the lowest number of control signals of all configurations, it achieves the highest throughput at a given transmission rate. This verifies the effectiveness of multi-beam massive MIMO, which can effectively utilize the transmission rate in the PHY layer.
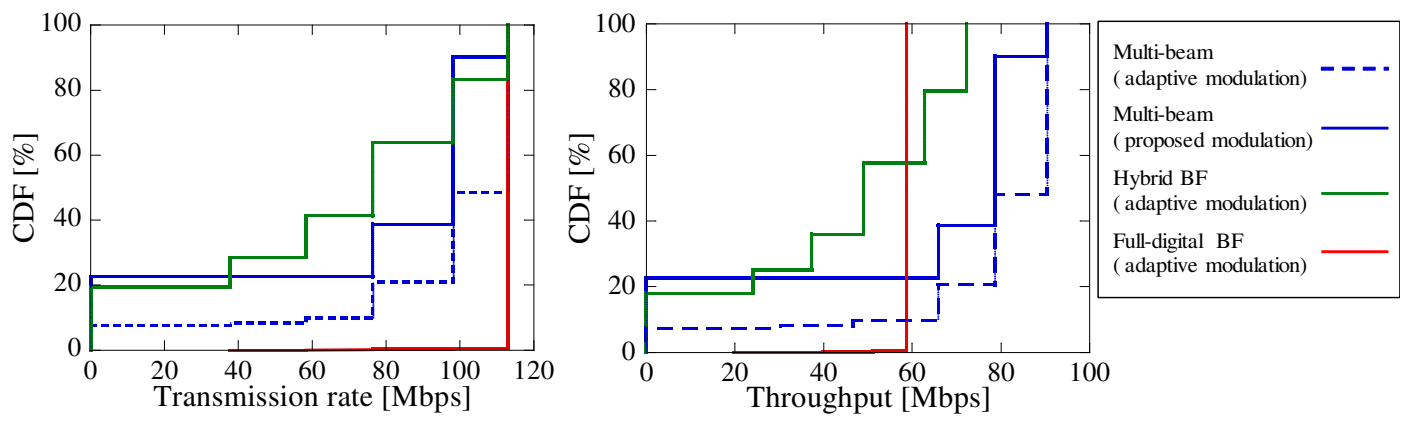

Figure 9. Characteristics of transmission rate and throughput under FDD-LTE.

\section{Conclusions}

In this paper, we proposed a simple adaptive modulation method for multi-beam massive MIMO and evaluated its performance when implemented in a MAC layer. The effectiveness of the proposed transmission method was validated through computer simulation. The performance of the method asymptotically approached the ideal characteristic because of the implementation of simple adaptive modulation using amplitude correlation and received power. The proposed method was also shown to achieve higher throughput characteristics compared to a hybrid approach with sub-arrays and a full-digital beam-forming configuration under the IEEE802.11ac and FDD-LTE environments. Future works include the further theoretical analysis and experimental evaluation using the analog multi-beam by Butler matrix circuit.

Author Contributions: This study was led by F.M. while K.N., R.T., T.H. and T.M. assisted with the computer simulations. 
Funding: This work was partially supported by the SCOPE \#165004002,\#185004002 and KAKENHI, Grant-in-Aid for Scientific Research (B) (17H01738, 17H03262).

Conflicts of Interest: The authors declare no conflict of interest.

\section{References}

1. Papadopoulos, H.; Wang, C.; Bursalioglu, O.; Hou, X.; Kishiyama, Y. Massive MIMO technologies and challenges towards 5G. IEICE Trans. Commun. 2015, E99-B, 602-621. [CrossRef]

2. Obara, T.; Suyama, S.; Shen, J.; Okumura, Y. Joint Processing of Analog Fixed Beamforming and CSI-Based Precoding for Super High Bit Rate Massive MIMO Transmission Using Higher Frequency Bands. IEICE Trans. Commun. 2015, E98-B, 1474-1481. [CrossRef]

3. Foschini, G.J.; Gans, M.J. On limits of wireless communications in a fading environment when using multiple antennas. Wirel. Pers. Commun. 1998, 6, 331-335. [CrossRef]

4. $\quad$ Gesbert, D.; Kountouris, M.; Heath, R.W., Jr.; Chae, C.B. ; Salzer, T. Shifting the MIMO paradigm. IEEE Signal Process. Mag. 2007, 24, 36-46. [CrossRef]

5. IEEE P802.11ac./D5.0, Part 11: Wireless LAN Medium Access Control (MAC and Physical Layer (PHY) Specifications; IEEE Standard for Information Technology; IEEE: New York, NY, USA, 2013.

6. 3rd Generation Partnership Project (3GPP). TS 36.213 Physical Layer Procedures; V10.2.0; 3GPP: Sophia Antipolis, France, 2018.

7. Lu, L.; Li, G.Y.; Swindlehurst, A.L.; Ashikhmin, A.; Zhang, R. An overview of massive MIMO: Benefits and challenges. IEEE J Sel. Top. Signal Process. 2014, 8, 742-758. [CrossRef]

8. Spencer, Q.H.; Peel, C.B.; Swindlehurst, A.L.; Haardt, M. An introduction to the multi-user MIMO downlink. IEEE Commun. Mag. 2004, 42, 60-67. [CrossRef]

9. Murakami, T.; Fukuzono, H.; Takatori, Y.; Mizoguchi, M. Multiuser MIMO with implicit channel feedback in massive antenna systems. IEICE Commun. Express 2013, 2, 336-342. [CrossRef]

10. Hiraguri, T.; Nishimori, K. Survey of transmission methods and efficiency using MIMO technologies for wireless LAN systems. IEICE Trans. Commun. 2015, E98-B, 1250-1267. [CrossRef]

11. Kameyama, K.; Nishimori, K.; Hiraguri, T.; Yamada, H.; Makino, H. Analog multi-beam massive MIMO transmission eliminating CSI estimation. IEICE Trans. Commun. 2016, J99-B, 743-752. (In Japanese)

12. Taniguchi, R.; Nishimori, K.; Makino, H. Multi-beam massive MIMO using constant modulus algorithm for QAM signals employing amplitude and phase offset compensation. IEICE Trans. Commun. 2017, E100-B, 262-268. [CrossRef]

13. Muramatsu, F.; Nishimori, K.; Taniguchi, R.; Hiraguri, T. Multi-beam massive MIMO with beam-selection using only amplitude information in uplink channel. IEICE Trans. Commun. 2018, E101-B, 1544-1551. [CrossRef]

14. Treichler, J.R.; Agee, B.G. A new approach to multipath correction of constant modulus signals. IEEE Trans. Acoust. Speech Signal Process. 1983, 31, 459-472. [CrossRef]

15. Agee, B. The least-Square CMA: A new technique for rapid correction of constant modulus signals. Proc. IEEE ICASSP 1986, 953-956.

16. Hyvarinen, A. Fast and robust fixed-point algorithms for independent component analysis. IEEE Trans. Neural Netw. 1999, 10, 626-634. [CrossRef]

17. Sohrabi, F.; Yu, W. Hybrid digital and analog beamforming design for large-scale antenna arrays. IEEE J. Sel. Top. Signal Process. 2016, 10, 501-513. [CrossRef]

18. Bogale, T.E.; Le, L.B. Beamforming for multiuser massive MIMO systems: Digital versus hybrid analog-digital. In Proceedings of the 2014 IEEE Global Communications Conference, Austin, TX, USA, 8-12 December 2014; pp. 4066-4071.

19. Molisch, A.F.; Ratnam, V.; Han, S.; Li, Z.; Nguyen, L.H.; Li, L.; Haneda, K. Hybrid beamforming for massive MIMO: A survey. IEEE Commun. Mag. 2017, 55, 134-141. [CrossRef]

20. Ngo, H.Q.; Larsson, E.G.; Marzetta, T.L. Energy and spectral efficiency of very large multiuser MIMO systems. IEEE Trans. Commun. 2013, 61, 1436-1449.

21. Rusek, F.; Persson, D.; Lau, B.K.; Larsson, E.G.; Marzetta, T.L.; Edfors, O.; Tufvesson, F. Scaling up MIMO: Opportunities and challenges with very large arrays. IEEE Signal Process. Mag. 2013, 30, 40-60. [CrossRef] 
22. Hoydis, J.; Brink, S.T.; Debbah, M. Massive MIMO in the UL/DL of cellular networks: How many antennas do we need? IEEE J. Sel. Areas Commun. 2013, 31, 160-171. [CrossRef]

23. Butler, J.; Lowe, R. Beamforming matrix simplifies design of electronically scanned antennas. Electron. Des. 1961, 9, 170-173.

24. Yamamoto, S.; Hirokawa, J.; Ando, M. A single-layer hollow-waveguide 8-way Butler matrix with modified phase shifters. In Proceedings of the ISAP2015, Hobart, Australia, 9-12 November 2015; pp. 937-940.

25. Zarzoso, V.; Comon, P. Robust independent component analysis by iterative maximization of the kurtosis contrast with algebraic optimal step size. IEEE Trans. Neural Netw. 2010, 21, 248-261. [CrossRef]

26. Gesbert, D.; Bolcskei, H.; Gore, D.A.; Paulraj, A.J. Outdoor MIMO wireless channels: Models and performance prediction. IEEE Trans. Commun. 2002, 50, 1926-1934. [CrossRef]

27. Oestges, C.; Erceg, V.; Paulraj, A.J. A physical scattering model for MIMO macrocellular broadband wireless channels. IEEE J. Sel. Areas Commun. 2003, 21, 721-729. [CrossRef]

28. Chung, S.T.; Goldsmith, A.J. Degrees of freedom in adaptive modulation: A unified view. IEEE Trans. Commun. 2001, 49, 1561-1571. [CrossRef]

29. Nishimori, K.; Yomo, H.; Popovski, P. Distributed interference cancellation for cognitive radios using periodic signals of the primary system. IEEE Trans. Wirel. Commun. 2011, 10, 2791-2981. [CrossRef]

30. Kikuma, N.; Nishimori, K.; Hiraguri, T. Effect of user antenna selection on block beamforming algorithms for suppressing inter-user interference in multiuser MIMO system. IEICE Trans. Commun. 2018, E101-B, 1523-1535. [CrossRef]

31. Tanbourgi, R.; Dhillon, H.S.; Andrews, J.G.; Jondral F.K. Effect of spatial interference correlation on the performance of maximum ratio combining. IEEE Trans. Wirel. Commun. 2014, 13, 3307-3316. [CrossRef]

32. Stankovic, V.; Haardt, M. Generalized design of multi-user MIMO precoding matrices. IEEE Trans. Wirel. Commun. 2008, 7, 953-961. [CrossRef]

33. Rathi, S.; Malik, N.; Chahal, N.; Malik, S. Throughput for TDD and FDD 4G LTE systems. Int. J. Innov. Technol. Explor. Eng. (IJITEE) 2014, 3, 73-77.

(C) 2019 by the authors. Licensee MDPI, Basel, Switzerland. This article is an open access article distributed under the terms and conditions of the Creative Commons Attribution (CC BY) license (http:// creativecommons.org/licenses/by/4.0/). 